\title{
Welche datenbasierten Servicemodelle sind erfolgsversprechend für KMU der Maschinen-, Anlagen-, Elektro- und Metallindustrie?
}

\author{
Helen Vogt $\mathbb{D} \cdot$ Matthias Ehrat $\cdot$ Rainer Fuchs $\cdot$ Manuel Holler
}

Eingegangen: 15. Dezember 2020 / Angenommen: 24. März 2021 / Online publiziert: 16. April 2021

(C) Der/die Autor(en) 2021

Zusammenfassung Durch die Möglichkeiten des Internet of Things (IoT) sind industrielle Produkte heute in der Lage, Anbieter und Kunden miteinander zu vernetzen. Produktbasierte Daten entstehen in allen Phasen des Produktlebenszyklus. Sie eröffnen den Anbietern die Möglichkeit, neue datenbasierte Servicemodelle zu entwickeln, um neuen Kundennutzen zu schaffen, der letztendlich durch neue digitale Geschäftsmodelle monetarisiert werden kann. Doch welche Ansätze bringen wirklichen Mehrwert für den Anbieter und sind damit den Implementierungsaufwand wert? Die vorliegende Studie untersucht, welche Servicemodelle für kleine und mittlere Unternehmen (KMU) der Maschinen-, Anlagen-, Elektro- und Metallindustrie erfolgversprechend sind. Es konnten zehn Servicemodelle identifiziert werden, die für die Unternehmen einen grundsätzlich hohen Mehrwert, jedoch teilweise unterschiedliche Implementierungsaufwände aufweisen. Die Vergütungsmodelle, mit denen die Servicemodelle bevorzugt monetarisiert werden können, erwiesen sich als vom Servicemodell selbst sowie von der Branche abhängig. In Summe wurde die Realisierungswahrscheinlichkeit für die datenbasierten Servicemodelle und die damit verbundenen digitalen Geschäftsmodelle innerhalb der nächsten drei Jahre noch als moderat eingeschätzt.

\footnotetext{
H. Vogt $(\bowtie) \cdot$ M. Ehrat $\cdot$ R. Fuchs $\cdot$ M. Holler Zurich University of Applied Sciences, Theaterstraße 17, 8400 Winterthur, Schweiz E-Mail: helen.vogt@zhaw.ch

M. Ehrat

E-Mail: matthias.ehrat@zhaw.ch

R. Fuchs

E-Mail: rainer.fuchs@zhaw.ch

M. Holler

E-Mail: manuel.holler@zhaw.ch
} 
Schlüsselwörter Industrie 4.0 · KMU · Digitale Geschäftsmodelle · Datenbasierte Servicemodelle · Vergütungsmodelle

\title{
What are the most promising data-based service models for SMEs in mechanical, plant, electrical and metal engineering?
}

\begin{abstract}
Given the possibilities of the Internet of Things (IoT), industrial products are now able to connect suppliers and customers. Product-based data are created at all stages of the product lifecycle. These data open up opportunities for suppliers to develop new data-based service models that create new customer benefits, which can ultimately be monetized through new digital business models. But what approaches add real value for the supplier and are thus worth the implementation effort? This study examines what service models are promising for small and medium-sized enterprises (SMEs) in the machinery, plant, electrical and metal industries. Ten service models were identified that offer companies a generally high added value, but differ partly in terms of their implementation efforts. The preferred revenue models with which the service models can be monetized were found to depend on the service model itself and on the industry. Overall, the probability of realization for the data-based service models and the associated digital business models within the next three years was assessed as still moderate.
\end{abstract}

Keywords Industry 4.0 $\cdot$ SME · Digital business models · Data-based service models $\cdot$ Revenue models

\section{Einleitung und Zielsetzung}

Im Zeitalter von Industrie 4.0 verändert sich das Umfeld von Unternehmen unaufhaltsam: Produkte, umfassend industrielle Erzeugnisse wie Maschinen, Anlagen, Geräte und Komponenten, werden ,smart und connected“ (Porter und Heppelmann 2014) und können dank Vernetzung im Internet of Things (IoT) in allen Lebensphasen Daten erzeugen (Fuchs und Barth 2018). Die Möglichkeiten, aus Nutzungsdaten neue Serviceangebote und Geschäftsmodelle zu entwickeln, sind enorm und verändern einige Branchen grundlegend (Wortmann et al. 2019), darunter besonders die produzierende Industrie (Porter und Heppelmann 2014). So befinden sich heute beispielsweise in den Produkten von Anlagenbauern zahlreiche Sensoren, die Daten über die Nutzung der Anlage beim Kunden erheben. Das so gewonnene Wissen versetzt den Anlagenbauer in die Lage, auf Basis der Daten zusätzliche Services anzubieten, die wiederum neue Ertrags- und Geschäftsmodelle ermöglichen.

Es zeigt sich jedoch, dass die Einführung von auf datenbasierten Services beruhenden, digitalen Geschäftsmodellen gerade im Anlagenbau nicht immer den erwünschten finanziellen Erfolg mit sich gebracht hat (Neely 2008; Davenport und Westerman 2018). Zwar haben einzelne Unternehmen mittlerweile eine systematische Herangehensweise an die Digitalisierung entwickelt, jedoch brauchen insbesondere kleine und mittlere Unternehmen (KMU) Unterstützung auf strategischer Ebene (Müller et al. 2018). Dies betrifft auch Unternehmen der Maschinen-, Anlagen-, 
Elektro- und Metallindustrie (MEM) ${ }^{1}$, die oftmals überfordert sind, die Nutzenpotentiale von Industrie 4.0 auszuschöpfen (Swissmem 2018). Es fehlen erfahrungsbasierte Handlungsempfehlungen, welche Ansätze sich in welchen Branchen bewährt haben und wie sie monetarisiert werden können.

In der vorliegenden Arbeit wird anhand einer empirischen Studie untersucht, welche datenbasierten Servicemodelle insbesondere für KMU der Branchenverbände Swissmem und VDMA, also der Maschinen-, Anlagen-, Elektro- und Metallindustrie, erfolgversprechend sind. Als Kriterien für die Attraktivität werden dabei einerseits das finanzielle resp. wirtschaftliche Potenzial, andererseits die realistische Umsetzbarkeit der Servicemodelle herangezogen. Zudem wird erläutert, mit welchen Vergütungsmodellen die datenbasierten Servicemodelle monetarisiert werden können. Es wird somit ein Beitrag zur systematischen Schaffung neuer digitaler Geschäftsmodelle für KMU der betrachteten Industrien erbracht.

\section{Digitale Geschäftsmodelle}

\subsection{Konzeptualisierung von Geschäftsmodellen}

Ein Geschäftsmodell beschreibt grundsätzlich, wie der Geschäftserfolg von Unternehmen zustande kommt. Es existieren dabei verschiedene Ansätze, wie Geschäftsmodelle dargestellt werden, wie beispielsweise der St. Galler Business Model Navigator (Gassmann et al. 2013), der Business Model Canvas (Osterwalder und Pigneur 2010) oder auch das Geschäftsmodellraster von Schallmo (2013). Im Wesentlichen werden in allen Darstellungsansätzen für Geschäftsmodelle vier grundsätzliche Fragen beantwortet: (1) Wer sind die Zielkunden, (2) was ist das Nutzenversprechen an diese Zielkunden, (3) wie kommt das Nutzenversprechen entlang der Wertschöpfungskette zustande und (4) mit welcher Ertragsmechanik verdient das Unternehmen Geld (Gassmann et al. 2013). Digitale Geschäftsmodelle zeichnen sich nach Jaekel (2015) in der Wertschöpfungsarchitektur durch ihren Fokus auf Daten und Analytik aus.

Im Rahmen dieser Arbeit wird insbesondere auf die Änderung des Nutzenversprechen durch die Einführung von datenbasierten Servicemodellen (siehe Frage 2) sowie auf die aus Sicht der Hersteller optimalen Ertragsmechanik resp. Vergütungsmodelle (Frage 4) für solche Servicemodelle eingegangen.

\subsection{Veränderung des Wertangebots durch datenbasierte Servicemodelle}

Im Rahmen von Industrie 4.0-Initiativen haben viele produzierende Unternehmen die Vorteile von IoT genutzt, um ihre internen Prozesse zu digitalisieren und so ihre Produktion und Logistik zu optimieren, was sich unter dem Begriff „Smart Facto-

\footnotetext{
1 Der Begriff MEM-Industrie entstammt der Begriffsdefinition des Schweizerischen Branchenverbandes Swissmem und ist äquivalent zur Definition des Maschinen- und Anlagenbaus des Verbandes Deutscher Maschinen- und Anlagenbau (VDMA). In dieser Arbeit wird der Begriff MEM als Sammelbegriff für die Maschinen-, Anlagen-, Elektro- und Metallindustrie verwendet.
} 
ry“ zusammenfassen lässt (BMWi 2018). Dank den Möglichkeiten des IoT können Unternehmen jedoch darüber hinaus auch ihr Wertangebot verändern, um sich im Markt durch zusätzlichen Kundennutzen in der Form von Mehrwertservices und neuen Vergütungsmodellen zu differenzieren (Bauernhansl et al. 2015; Rymaszewska et al. 2017; Wortmann et al. 2019). In diesem Zusammenhang wird IoT als die Ausstattung von Geräten mit Sensoren verstanden, so dass die Geräte die Fähigkeit erhalten, Daten zu sammeln und diese zu kommunizieren (Rymaszewska et al. 2017) und als autonome Teilnehmer in das Internet integriert zu werden (Schneider 2016).

Industrieunternehmen weiten dabei ihre Wertschöpfungskette um die Bereitstellung datenbasierter Servicemodelle aus und ändern die Logik, wie die verschiedenen Komponenten der Wertschöpfung monetarisiert werden (Rymaszewska et al. 2017). Die Mechanik der digitalen Geschäftsmodelle für datenbasierte Servicemodelle fügt sich hierbei in die verwandten Frameworks der Servitization (Roy et al. 2009) und der Product-Service-Systeme (PSS) (Baines et al. 2007) ein. Beide Konzepte beschreiben, wie Industriefirmen die Wertschöpfung weg vom Verkauf eines Produktes (Maschinen, Anlagen, Geräte und Komponenten) hin zum Verkauf eines Systems, bestehend aus Produkt und margenstarken Services, verschieben (Lightfoot et al. 2013). Die Basis hierbei bildet das physische Produkt, welches im Feld betrieben wird. Mittels eingebauter Sensoren sammelt das Produkt Zustands-, Bewegungs- und Nutzungsdaten und speichert diese über eine Anbindung ans Internet typischerweise in cloud-basierten Datenspeichern (Vogel-Heuser 2017). Diese Daten können durch den Hersteller ausgewertet und wahlweise mit externen Daten angereichert werden, um daraus datenbasierte Servicemodelle zu entwickeln (Acatech 2014). Solche Servicemodelle finden in unterschiedlichen Phasen des Produktlebenszyklus Anwendung. Sie umfassen Angebote wie z. B. das nutzungsbasierte Konfigurationsdesign, die Zustandsüberwachung der Anlage, Ferndiagnose und präventive Wartung, Prozessparametrierung und -optimierung, Verfügbarkeitsverbesserung, Qualitätsmanagement und mehr (Paukstadt et al. 2019).

Durch die Einführung datenbasierter Servicemodelle erhalten Industriefirmen mehr Transparenz über den Nutzen ihrer Produkte im Feld, können ihre Umsätze und Gewinne steigern sowie die Kundenbeziehung auch nach dem Verkauf aufrechterhalten und vertiefen (Haller et al. 2008; Gerpott und May 2016; Paukstadt et al. 2019).

\subsection{Systematik bei der Entwicklung von digitalen Geschäftsmodellen}

Obwohl digitale Geschäftsmodelle im Business-to Business (B2B)-Umfeld zunehmen, wird in mehreren Arbeiten festgestellt, dass sich der finanzielle Erfolg oft nicht unmittelbar einstellt (Neely 2008; Davenport und Westerman 2018). Auch die regelmäßig durch Swissmem durchgeführte Umfrage zum Umsetzungsstand von Industrie 4.0 in der Schweiz kommt zu diesem Schluss (Swissmem 2018). Es fehlen gerade für KMU Erkenntnisse und Handlungsempfehlungen (Müller et al. 2018), um datenbasierte Servicemodelle und somit digitale Geschäftsmodelle zu identifizieren, die tatsächlich Nutzen generieren und für Unternehmen auch realistisch umsetzbar sind. Eine wachsende Anzahl wissenschaftlicher Publikationen beschreibt Ansätze, wie produzierende Unternehmen ihre Geschäftsmodelle durch die Anbindung von 
digitalen Leistungskomponenten erweitern können (Fleisch et al. 2015; Scherrer et al. 2017; Suppatvech et al. 2019). Je nach Publikationen werden verschiedene datenbasierte Servicemodelle genannt. Im Folgenden wird beschrieben, wie die genannten Ansätze auf ihre Relevanz für KMU in der MEM-Industrie untersucht und hinsichtlich Mehrwert, Implementierungsaufwand, Realisierungswahrscheinlichkeit und Monetarisierbarkeit eingeordnet wurden.

\section{Empirische Studie}

\subsection{Studiendesign}

Datenbasierte Servicemodelle und damit verbundene digitale Geschäftsmodelle bergen unterschiedliche Erfolgspotentiale, aber auch substanzielle Hürden bei der Umsetzung (Friedli und Gebauer 2003; Oliva und Kallenberg 2003; Krebs et al. 2017). Wenig diskutiert wurde bisher, welche der potenziellen Servicemodelle das größte ökonomische Potenzial für produzierende Firmen aus dem B2B-Umfeld bieten. Um diese Frage zu adressieren, wurde ein Mixed-Methods-Ansatz gewählt. Das methodische Vorgehen für die Literaturrecherche, die qualitative und quantitative Erhebung sowie die Beschreibung der jeweiligen Stichprobe werden in Tab. 1 zusammengefasst.

\subsection{Studiendurchführung}

Zunächst wurde eine Literaturrecherche durchgeführt, um datenbasierte Servicemodelle zu identifizieren, welche auf Nutzungsdaten von industriellen Produkten wie Maschinen, Anlagen, Geräte und Komponenten basieren. Die folgenden Leitfragen gaben den Rahmen:

- Welche datenbasierte Servicemodelle gibt es und mittels welcher Vergütungsmodelle werden diese im Rahmen von digitalen Geschäftsmodellen monetarisiert?

- Welche dieser Servicemodelle eignen sich im Besonderen für KMU der MEMBranche?

Aufgrund dieser Leitfragen wurden Suchbegriffe entwickelt und in fünf verschiedenen Datenbanken (ABI/INFORM Global, Emerald, Springer, Google Scholar, Science Direct) abgefragt. Die ausgewählten Artikel wurden mittels Volltextanalyse bewertet und thematisch gruppiert. Basierend darauf wurden 14 datenbasierte Servicemodelle sowie vier Vergütungsmodelle (Abschn. 4.1) identifiziert.

Im zweiten Schritt wurden mittels einer qualitativen Befragung die Anforderungen aus der Industrie an die Servicemodelle erhoben sowie deren Eignung für den Einsatz bei KMU bewertet. Die 14 Servicemodelle wurden mit acht Industrievertretern sowie vier Branchenexperten mittels qualitativer Interviews evaluiert. Es fand eine Bewertung der Modelle hinsichtlich des erwarteten Mehrwertes sowie des erwarteten Implementierungsaufwandes statt. Im Weiteren wurde die Wahrscheinlichkeit der Realisierung der Servicemodelle innerhalb der nächsten drei Jahre abgefragt. 
Tab. 1 Übersicht des Studiendesigns

\begin{tabular}{|c|c|c|c|}
\hline & Literaturrecherche & Qualitative Erhebung & Quantitative Erhebung \\
\hline $\begin{array}{l}\text { Ziel- } \\
\text { setzung }\end{array}$ & $\begin{array}{l}\text { Identifikation } \\
\text { Stand des Wis- } \\
\text { sens zu datenba- } \\
\text { sierten Service- } \\
\text { und Vergütungs- } \\
\text { modellen }\end{array}$ & $\begin{array}{l}\text { Bewertung der in der Litera- } \\
\text { turrecherche identifizierten } \\
\text { Servicemodelle hinsichtlich } \\
\text { ihrer Eignung für KMU und } \\
\text { Eliminierung von nicht geeig- } \\
\text { neten Modellen }\end{array}$ & $\begin{array}{l}\text { Bewertung von Mehrwert, Imple- } \\
\text { mentierungsaufwand und Reali- } \\
\text { sierungswahrscheinlichkeit sowie } \\
\text { Identifizierung der favorisierten } \\
\text { Vergütungsmodelle für die ausge- } \\
\text { wählten Servicemodelle }\end{array}$ \\
\hline $\begin{array}{l}\text { Erhebungs- } \\
\text { methode }\end{array}$ & $\begin{array}{l}\text { Datenbank- } \\
\text { Recherche }\end{array}$ & $\begin{array}{l}\text { Qualitative Interviews (per- } \\
\text { sönlich oder telefonisch) mit } \\
\text { teilstrukturiertem Fragebogen }\end{array}$ & $\begin{array}{l}\text { Online-Umfrage mit Google } \\
\text { Forms }\end{array}$ \\
\hline $\begin{array}{l}\text { Erhebungs- } \\
\text { zeitraum }\end{array}$ & $\begin{array}{l}01.04 .2019- \\
30.05 .2019\end{array}$ & 05.06.2019-30.06.2019 & 23.10.2019-25.11.2019 \\
\hline $\begin{array}{l}\text { Anzahl } \\
\text { Befra- } \\
\text { gungen }\end{array}$ & n.a. & 12 Interviews & 192 Online Befragungen \\
\hline $\begin{array}{l}\text { Stichpro- } \\
\text { benselek- } \\
\text { tion }\end{array}$ & n.a. & $\begin{array}{l}\text { Manuelle Rekrutierung von } \\
\text { Produktmanagern, Fach- } \\
\text { spezialisten und Marketing- } \\
\text { verantwortlichen von Schwei- } \\
\text { zer Unternehmen aus der } \\
\text { MEM-Branche }\end{array}$ & $\begin{array}{l}\text { Selektion von Unternehmen aus } \\
\text { der MEM-Branche mit Hauptsitz } \\
\text { in der DACH }- \text { Region aus dem } \\
\text { Swissmem- und VDMA-Verzeich- } \\
\text { nis sowie der Datenbank Orbis }\end{array}$ \\
\hline $\begin{array}{l}\text { Auswer- } \\
\text { tungsme- } \\
\text { thode }\end{array}$ & $\begin{array}{l}\text { Vorauswahl } \\
\text { Zusammenfassende } \\
\text { Inhaltsanalyse }\end{array}$ & $\begin{array}{l}\text { Transkription } \\
\text { Zusammenfassende Inhalts- } \\
\text { analyse }\end{array}$ & $\begin{array}{l}\text { Deskriptive Statistik } \\
\text { Signifikanzanalyse }\end{array}$ \\
\hline
\end{tabular}

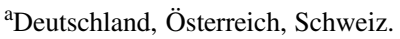

Tab. 2 Übersicht der ausgewählten Servicemodelle

\begin{tabular}{|c|c|}
\hline Servicemodell & Beschreibung \\
\hline vider & $\begin{array}{l}\text { Nebst der Maschine/Anlage werden zusätzlich datenbasierte Systemkonfiguratio- } \\
\text { nen, Installations- und Engineering Services angeboten }\end{array}$ \\
\hline Benchmarking & $\begin{array}{l}\text { Die Produktionsleistung von Maschinen/Anlagen wird erfasst, damit der Kunde } \\
\text { die Anlagen firmenintern vergleichen oder mit anderen Firmen benchmarken kann }\end{array}$ \\
\hline $\begin{array}{l}\text { Qualitätsmanage- } \\
\text { ment }\end{array}$ & $\begin{array}{l}\text { Produkt-/Produktionsdaten werden erfasst, damit der Kunde die Rückverfolgbar- } \\
\text { keit, Produktqualität und Compliance sicherstellen kann }\end{array}$ \\
\hline Ersatzteilanzeige & $\begin{array}{l}\text { Die Maschine/Anlage zeigt bei Verschleiß oder Bedarf das notwendige Ersatzteil } \\
\text { automatisch an, damit die korrekten Ersatzteile bestellt werden können }\end{array}$ \\
\hline $\begin{array}{l}\text { Object Self- } \\
\text { Service }\end{array}$ & $\begin{array}{l}\text { Die Maschine/Anlage überwacht das Verbrauchsmaterial und löst bei Bedarf auto- } \\
\text { nom eine Bestellung aus oder generiert einen Bestellvorschlag }\end{array}$ \\
\hline Digital Add-on & $\begin{array}{l}\text { Die Maschine/Anlage wird mit Basisfunktionen verkauft; neue Funktionen werden } \\
\text { via Software hinzugefügt resp. freigeschaltet }\end{array}$ \\
\hline $\begin{array}{l}\text { Anlagenmanage- } \\
\text { ment }\end{array}$ & $\begin{array}{l}\text { Die Maschine/Anlage generiert eine Warnanzeige und/oder passt die Anlagenleis- } \\
\text { tung dem aktuellen Anlagenzustand an }\end{array}$ \\
\hline Uptime-Garantie & $\begin{array}{l}\text { Dem Kunden wird über einen definierten Zeitraum oder Leistungsumfang eine } \\
\text { spezifizierte Anlagenverfügbarkeit garantiert }\end{array}$ \\
\hline Ferndiagnose & $\begin{array}{l}\text { Die Maschine/Anlage generiert bei Problemen automatisierte Fehlermeldungen. } \\
\text { Der Hersteller bietet einen Online-Support an }\end{array}$ \\
\hline Plattform-Modell & $\begin{array}{l}\text { Eine Plattform verbindet Datenlieferanten mit -nutzern. Sensordaten werden über } \\
\text { diese Plattform zur Verfügung gestellt resp. verkauft }\end{array}$ \\
\hline
\end{tabular}




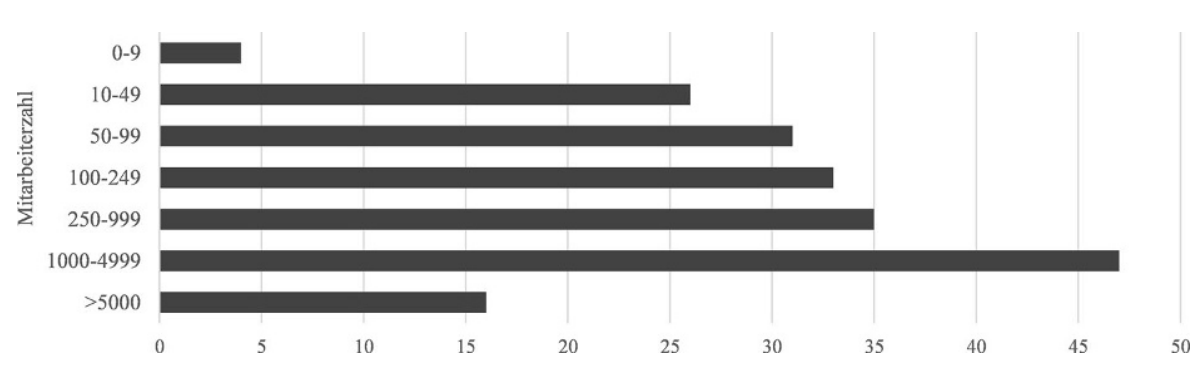

Abb. 1 Verteilung der Datensätze nach Unternehmensgrößen, $N=192$

Auf der Basis dieser Bewertung durch die Experten und Industrievertreter wurden vier Servicemodelle aus der ursprünglichen Liste eliminiert.

Im dritten Schritt wurden die verbleibenden zehn Servicemodelle (siehe Tab. 2) in einer quantitativen Erhebung bei mittelständischen Firmen aus der MEM-Branche in der DACH-Region nochmals hinsichtlich des erwarteten Mehrwertes, Implementierungsaufwandes und Realisierungswahrscheinlichkeit bewertet. Die Teilnehmer haben dies anhand einer Skala von 1 (sehr niedrig) bis 6 (sehr hoch) festgemacht. Zudem wurde abgeschätzt, mittels welcher Vergütungsmodelle die jeweiligen Servicemodelle monetarisierbar sein könnten. Dazu haben die Teilnehmer aus den vier Vergütungsmodellen die für sie wichtigsten Modelle (max. zwei) pro Servicemodell identifiziert.

Für die Identifizierung von relevanten Unternehmen der MEM-Branche wurden die Mitgliederverzeichnisse des Swissmem ${ }^{2}$ und des VDMA ${ }^{3}$ verwendet sowie eine Abfrage aus der Datenbank Orbis mit den NACE-Codes ${ }^{4}$ 2410-2899 erstellt. Es wurden nur Firmen aus der DACH-Region berücksichtigt. Der Schwerpunkt lag auf größeren Unternehmen, da eine systematische Entwicklung von datenbasierten Servicemodellen erst ab einer gewissen Unternehmensgröße zu erwarten ist. Insgesamt wurden 192 Unternehmensvertreter befragt (Abb. 1).

Von den 192 Studienteilnehmern stammen 182 Unternehmen aus dem Kernbereich der MEM-Branche. Davon ordnet sich die Mehrheit, nämlich 81 Unternehmen, dem Maschinenbau zu. 38 der befragten Unternehmen sind im Anlagenbau inklusive der Realisierung von Systemlösungen tätig. 50 Unternehmen stellen Geräte, elektrische Ausrüstung und Komponenten her. Wir fassen diese Unternehmen in der Kategorie „Gerätebau und Komponenten“ zusammen. Weitere 13 Unternehmen sind in der Metallerzeugung und -bearbeitung tätig. Außerhalb des Kernbereiches der MEM-Branche haben sich weitere 10 Unternehmen mehrheitlich aus dem Automobilbau an der Studie beteiligt, die von den branchenspezifischen Auswertungen ausgeschlossen wurden. Der Großteil der Firmen (78) betreibt Serienfertigung, 64 haben Einzel-, 35 haben Sorten- und 13 Unternehmen haben Massenfertigung, zwei weitere wurden unter „Sonstiges“ klassifiziert. Von den 192 befragten Unter-

\footnotetext{
2 https://www.swissmem.ch/de/verband/mitgliederverzeichnis.html.

3 https://www.vdma.org/mitglieder.

4 Nomenclature statistique des activités économiques dans la Communauté européenne resp. Statistische Systematik der Wirtschaftszweige in der Europäischen Gemeinschaft.
} 
nehmen entwickeln 125 die Software ihrer Produkte selbst, 36 beziehen die Software für ihre Produkte von Dritten und 31 benötigen gegenwärtig noch keine Software für die Nutzung ihrer Produkte.

\section{Ergebnisse}

\subsection{Resultate der Literaturrecherche sowie der qualitativen Erhebung}

Basierend auf der Literaturrecherche (Gassmann et al. 2013; Fleisch et al. 2015; Herterich et al. 2015; Suppatvech et al. 2019) sowie den Experteninterviews wurden zehn Servicemodelle (Tab. 2) als relevant für KMU identifiziert.

In der Literaturrecherche wurden zudem die folgenden drei resp. vier Ertragsmechanismen resp. Vergütungsmodelle identifiziert (Tab. 3).

\subsection{Resultate der quantitativen Erhebung}

\subsubsection{Branchenübergreifende Bewertung der abgefragten Servicemodelle}

Allen zehn in der qualitativen Erhebung identifizierten Servicemodellen wurde von den Befragten in der quantitativen Studie ein moderater bis hoher Mehrwert für die anbietenden Unternehmen bescheinigt. Unter diesem Mehrwert ist nicht nur der reine finanzielle Nutzen zu verstehen, sondern es zählen auch weitere wirtschaftliche Elemente wie Einfluss auf die Kundenbeziehung sowie Imagewirkung.

In Abb. 2 ist die Bewertung der Servicemodelle hinsichtlich des Mehrwertes und Implementierungsaufwandes dargestellt. Alle datenbasierten Servicemodelle erhielten auf der Skala von 1 bis 6 durchschnittlich Bewertungen von über 3,75 bis knapp 5. Besonders hoch wurde der potenzielle Mehrwert der Servicemodelle Ferndiagnose und Ersatzteilanzeige sowie des Solution Provider-Modells eingeschätzt. Für Letzteres wird jedoch der Implementierungsaufwand im Vergleich als relativ hoch angesehen.

Die OLS 5 -Ausgleichsgerade $(y=-0,4148 x+6,1664)$ verdeutlicht mit einem Bestimmtheitsmaß von $\mathrm{R}^{2}=0,262$, dass der Mehrwert eines Servicemodells im All-

Tab. 3 Übersicht der Vergütungsmodelle (basierend auf Suppatvech et al. 2019; Wortmann et al. 2019)

\begin{tabular}{ll}
\hline Vergütungsmodell & Beschreibung \\
\hline Einmalzahlung & $\begin{array}{l}\text { Einmalige Vergütung, die eine dauerhafte Nutzung der datenbasierten Services } \\
\text { ermöglicht }\end{array}$ \\
$\begin{array}{l}\text { Leistungs- bzw. } \\
\text { nutzungsabhängi- } \\
\text { ge Zahlung }\end{array}$ & $\begin{array}{l}\text { Der Preis hängt von der erbrachten Leistung (z. B. der Produktionsleistung, An- } \\
\text { lagenverfügbarkeit oder Produktqualität) bzw. von der Nutzungsdauer oder -häu- } \\
\text { figkeit (z. B. der Anzahl Transaktionen, verbrauchter/geleisteter Einheiten oder } \\
\text { Betriebsstunden) ab } \\
\text { Regelmäßige }\end{array}$ \\
$\begin{array}{l}\text { Periodische Zahlung für die Nutzung der datenbasierten Services für einen festge- } \\
\text { legten Zeitraum }\end{array}$ \\
\hline
\end{tabular}

\footnotetext{
5 Ordinary Least Squares (gewöhnliche Methode der kleinsten Quadrate).
} 


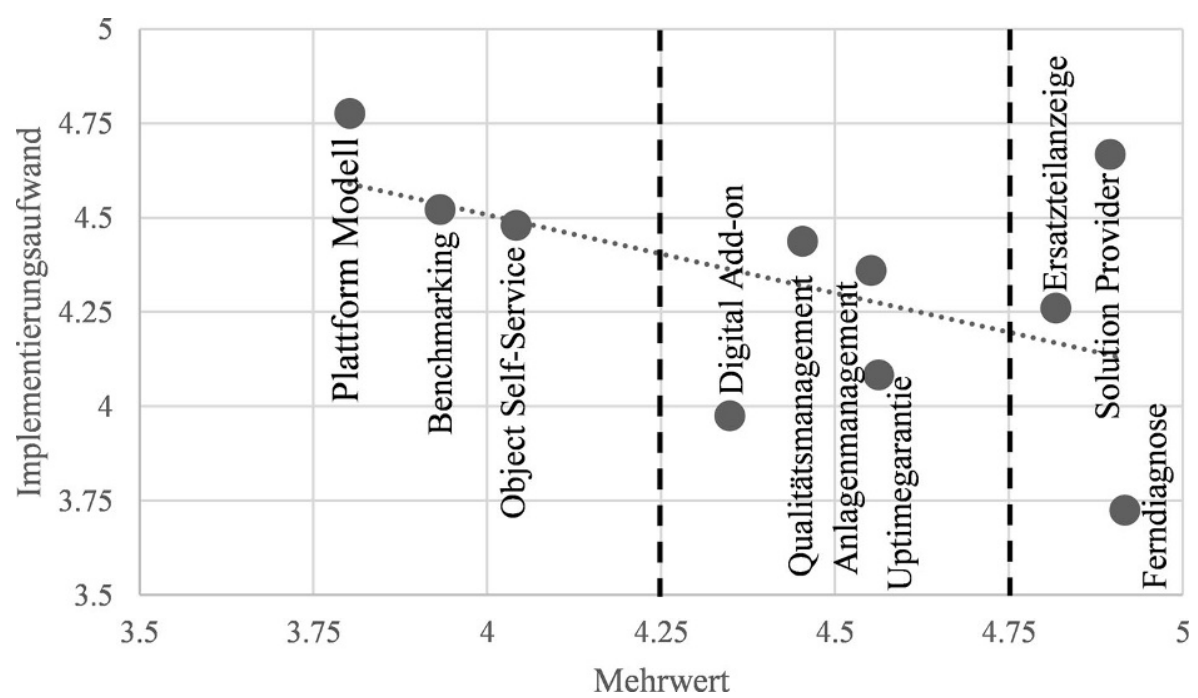

Abb. 2 Mehrwert für Unternehmen, die digitale Servicemodelle anbieten, in Relation zum Implementierungsaufwand der Unternehmen, $N=192$

gemeinen umso positiver beurteilt wird, je geringer der Implementierungsaufwand ist. Das Modell Solution Provider stellt in dieser Hinsicht einen Ausreißer im vereinfachten linearen Modell dar. Abb. 2 zeigt drei charakteristische Bereiche der Servicemodelle, welche durch gestrichelte Linien abgegrenzt sind. Der rechte Bereich umfasst Servicemodelle, die sich durch einen relativ hohen Mehrwert bei moderatem Implementierungsaufwand auszeichnen. Der mittlere Bereich umfasst Servicemodelle, welche bei grundsätzlichem Mehrwert einen nur unwesentlich höheren Implementierungsaufwand erfordern. Die Servicemodelle im linken Bereich verursachen einen zu hohen Implementierungsaufwand im Vergleich zum Mehrwert.

Die Angebote mit einem hohen Mehrwert und vertretbarem Implementierungsaufwand erweisen sich (Abb. 3) auch als diejenigen mit der höchsten Realisierungswahrscheinlichkeit innerhalb der nächsten drei Jahre. Die OLS-Ausgleichsgerade $(y=1,01 x-1,2989)$ verdeutlicht hier mit einem Bestimmtheitsmaß von $R^{2}=0,7233$, dass die Realisierungswahrscheinlichkeit umso grösser ist, je höher der erwartete Mehrwert ist. Mit einer durchschnittlichen Bewertung von 3,18 auf der Skala von 1 bis 6 wird die Realisierungswahrscheinlichkeit der Servicemodelle für die nächsten drei Jahre jedoch insgesamt als eher niedrig eingeschätzt.

Abb. 4 visualisiert die Einschätzung der Befragten hinsichtlich einer möglichen Monetarisierbarkeit der untersuchten Servicemodelle mit den verschiedenen Vergütungsmodellen. Im Durchschnitt über alle betrachteten Servicemodelle erachten $27 \%$ der Befragten eine regelmäßige, periodisch wiederkehrende Zahlung als geeignete Vergütungsform. Regelmäßige Zahlungen werden bei den Servicemodellen Qualitätsmanagement, Plattformmodell und Ferndiagnose als erfolgversprechend eingeschätzt. 29\% der Befragten erachten eine Einmalzahlung als geeignete Form der Vergütung. Prädestiniert für Einmalzahlungen sind dabei die Servicemodelle Solution Provider, Digital Add-on und Benchmarking. $44 \%$ der Befragten erwarten eine 


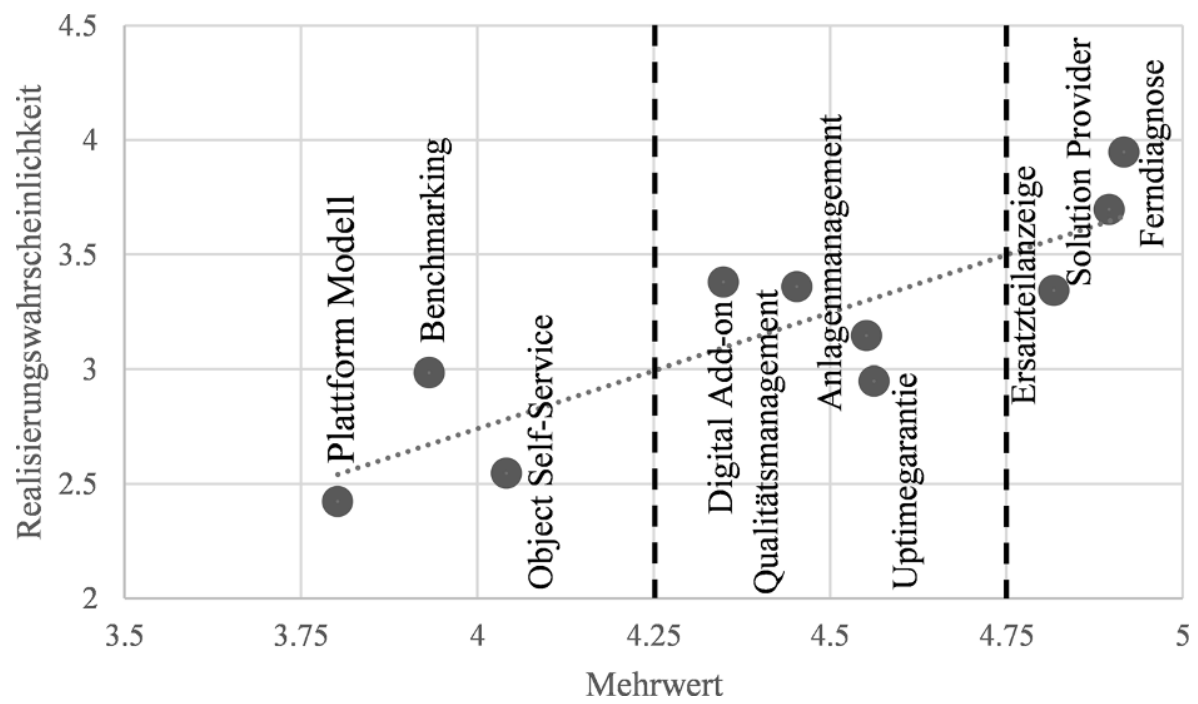

Abb. 3 Mehrwert für Unternehmen, die digitale Servicemodelle anbieten, in Relation zur erwarteten Realisierungswahrscheinlichkeit innerhalb der nächsten drei Jahre, $N=192$

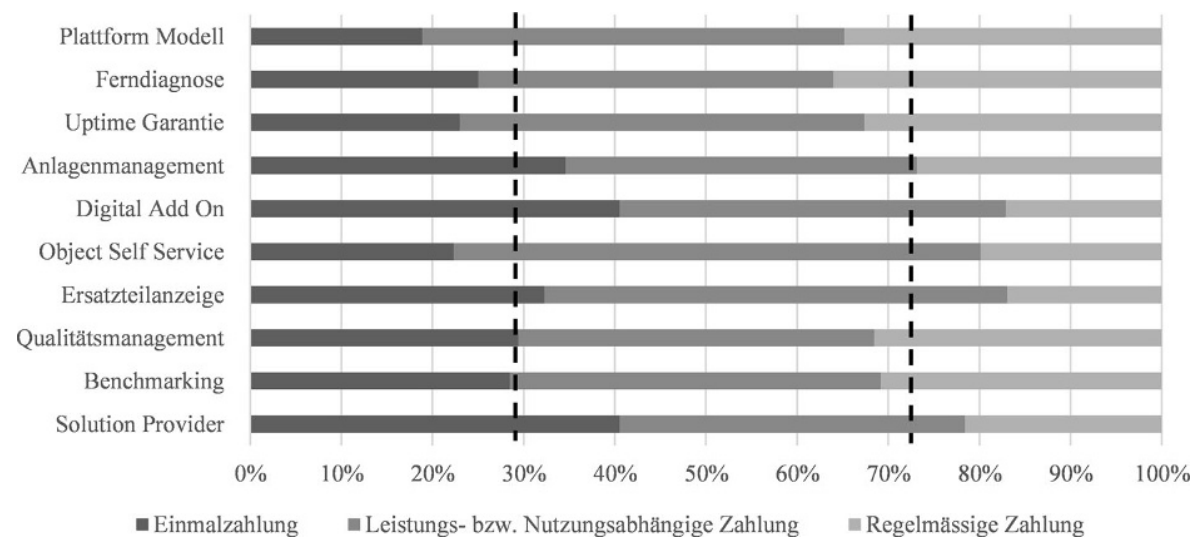

Abb. 4 Präferenz der Vergütungsmodelle für die untersuchten Servicemodelle, $N=192$

Präferenz für leistungs- und nutzungsabhängige Zahlungen. Insbesondere die Servicemodelle Object Self-Service und Ersatzteilanzeige erscheinen für eine leistungsbzw. nutzungsabhängige Zahlung prädestiniert.

\subsubsection{Branchenspezifische Unterschiede bei der Bewertung der abgefragten Servicemodelle}

Um Unterschiede hinsichtlich der Bewertung des Mehrwertes der Servicemodelle in Abhängigkeit der Branchenzugehörigkeit zu untersuchen, wurde eine Signifikanzanalyse durchgeführt. Hierbei zeigt sich, dass Unternehmen in den Branchen Metallerzeugung und -bearbeitung den Mehrwert aller Servicemodelle au- 


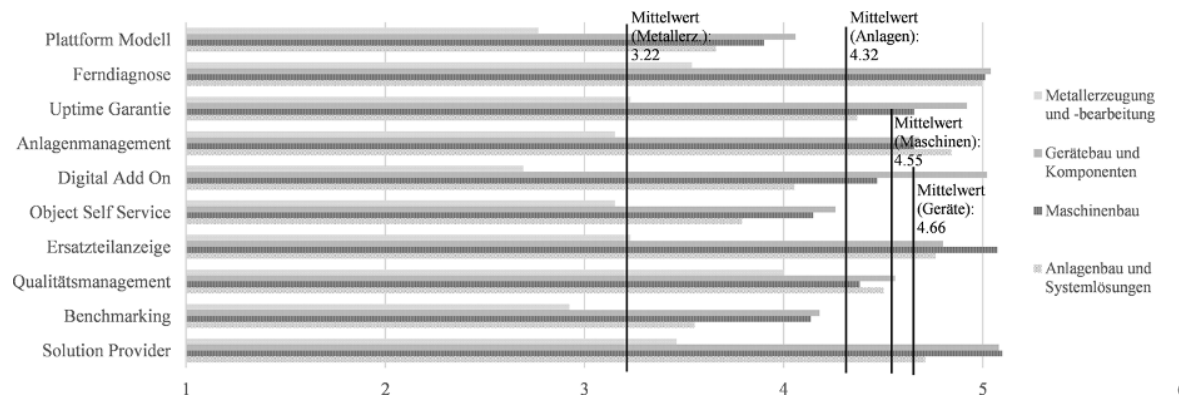

Abb. 5 Beurteilung des Mehrwerts der Servicemodelle aus Branchensicht, $N=182$

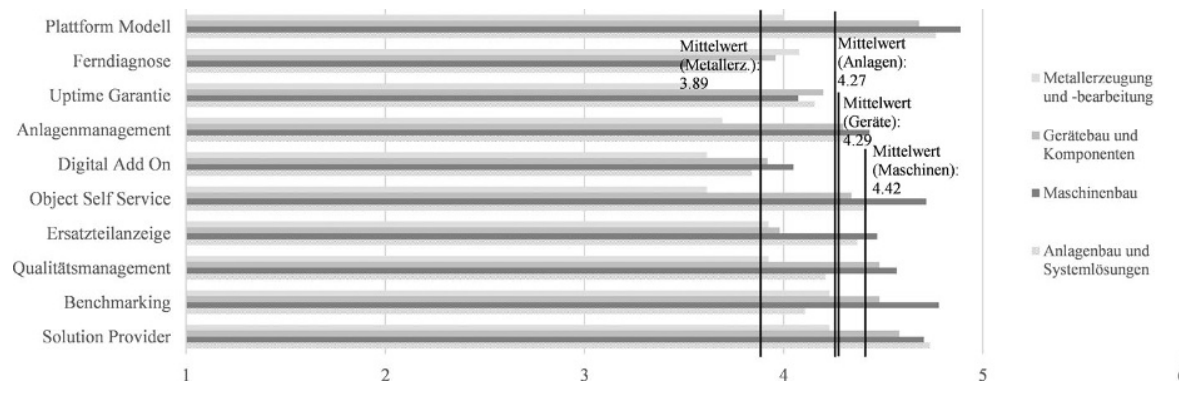

Abb. 6 Beurteilung des Implementierungsaufwands der Servicemodelle aus Branchensicht, $N=182$

Ber Qualitätsmanagement deutlich tiefer als Firmen aus anderen Branchen beurteilen. Exemplarisch kann dies für das Servicemodell Solution Provider aufgezeigt werden: Post-hoc-Tests mit Games-Howell-Korrektur zeigen, dass sich die Bewertung des Mehrwertes des Solution Provider-Modells bei Metallerzeugung und -bearbeitung $(M=3,46, S D=1,85)$ signifikant von den entsprechenden Bewertungen in den Branchen Maschinenbau sowie Gerätebau und Komponenten unterscheidet $(\mathrm{F}(5,186)=5,7, p<0,05)$.

In Abb. 5 zeigt sich, dass alle Branchen außer Metallerzeugung und -bearbeitung den Mehrwert der Servicemodelle jeweils sehr ähnlich bewerten. Die höchste Übereinstimmung wird beim Servicemodell Ferndiagnose ersichtlich, welches jeweils mit 5 bewertet wird. Ebenfalls eine hohe Bewertung erhalten die Servicemodelle Solution Provider und Ersatzteilanzeige.

Während die Unternehmen der Metallerzeugung und -bearbeitung den Mehrwert der Servicemodelle im Branchenvergleich am geringsten einschätzen, beurteilen sie auch den erforderlichen Implementierungsaufwand als am kleinsten (3,89, Abb. 6). Die übrigen Branchen ähneln sich stark hinsichtlich der Beurteilung des Implementierungsaufwandes. Die höchste Hürde stellt die Implementierung solcher Servicemodelle für die Maschinenindustrie dar $(4,42)$, gefolgt von den Branchen Gerätebau und Komponenten (4,29) sowie Anlagenbau und Systemlösungen (4,27). Der Maschinenbau beurteilt den Implementierungsaufwand für die meisten einzelnen Servicemodelle im Branchenvergleich am höchsten, lediglich die Ferndiagnose und 


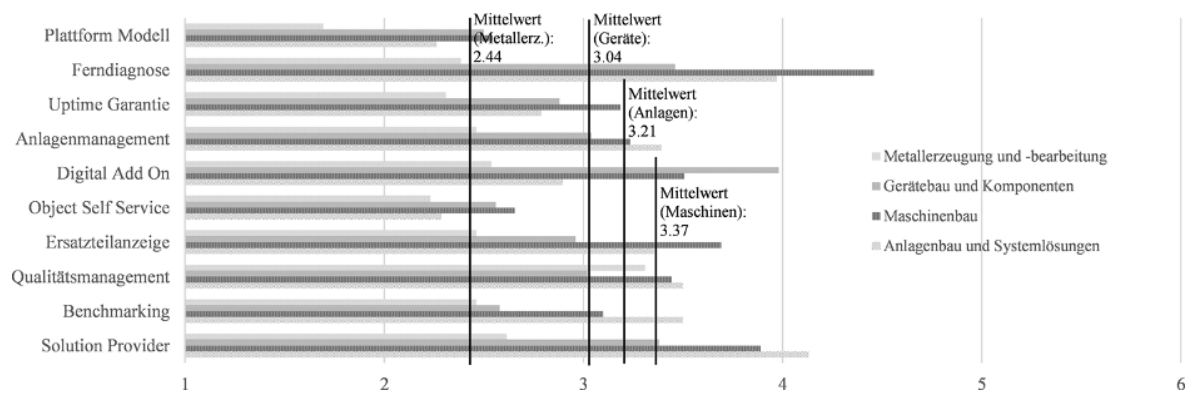

Abb. 7 Beurteilung der Realisierungswahrscheinlichkeit der Servicemodelle aus Branchensicht. $N=182$

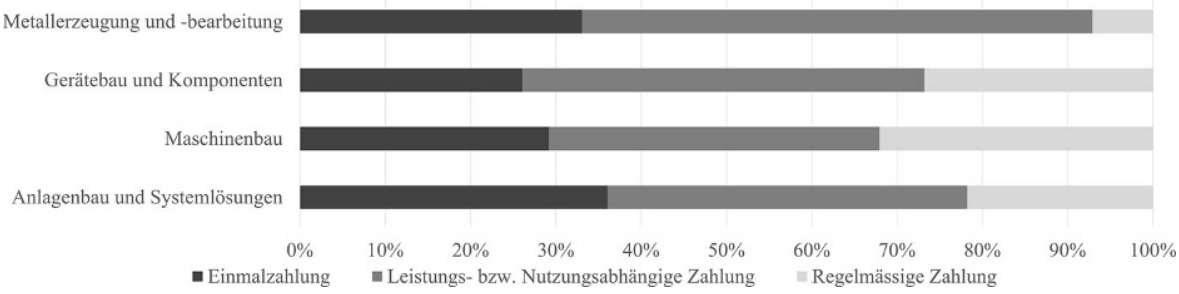

Abb. 8 Branchenspezifische Unterschiede bei der Präferenz der Vergütungsmodelle für die untersuchten Servicemodelle, $N=182$

Uptime-Garantie werden von Unternehmen des Anlagenbaus als noch aufwändiger beurteilt.

Auch hinsichtlich der Realisierungswahrscheinlichkeit der untersuchten datenbasierten Servicemodelle fallen die Unternehmen der Metallerzeugung und -bearbeitung mit einem Mittelwert von 2,44 deutlich ab (Abb. 7). Wieder liegen die anderen Branchen in der Beurteilung eng zusammen, mit Bewertungen zwischen 3,04 und 3,37 auf der Skala von 1 bis 6 sind sie im Durchschnitt jedoch eher zurückhaltend. Lediglich für die Servicemodelle Ferndiagnose und Solution Provider werden von mehr als einer Branche deutlich höhere Realisierungswahrscheinlichkeiten als der Durchschnitt aller Servicemodelle angegeben. In der Metallerzeugung und -bearbeitung sticht lediglich das Qualitätsmanagement heraus, das mit einem Wert von 3,25 eine deutlich höhere Realisierungswahrscheinlichkeit als der Durchschnitt in dieser Branche $(2,44)$ aufweist. Für die Branche Gerätebau und Komponenten weist das Modell Digital Add-on die höchste Realisierungswahrscheinlichkeit auf, einhergehend mit einem überdurchschnittlich hohen Mehrwert (Abb. 4) bei unterdurchschnittlichem Implementierungsaufwand (Abb. 5).

Hinsichtlich der Präferenz für Vergütungsmodelle (Abb. 8) ist festzustellen, dass $60 \%$ der Unternehmen der Metallindustrie eine leistungs- und nutzungsabhängige Zahlung bevorzugen, was klar über dem durchschnittlichen Wert von $43 \%$ über alle Branchen hinweg liegt. Regelmäßige Zahlungen sind für Unternehmen der Metallindustrie hingegen am wenigsten relevant; nur $7 \%$ würden solche bevorzugen $(27 \%$ über alle Branchen). Im Maschinenbau wird die regelmäßige Vergütung hingegen überdurchschnittlich oft bevorzugt (32\%). Leistungs- bzw. nutzungsabhängige Zahlungen werden hier im Vergleich zu den anderen Branchen weniger stark angestrebt. 


\section{Diskussion}

Die vorliegende Studie konnte verdeutlichen, dass KMU der Maschinen-, Anlagen-, Elektro- und Metallindustrie datenbasierte Servicemodelle sehr unterschiedlich bezüglich ihres Mehrwerts für die Unternehmen, aber auch in Bezug auf den erwarteten Aufwand für die Implementierung beurteilen.

Betrachtet man die Branchen im Detail, fällt auf, dass Unternehmen, die dem Maschinenbau zuzuordnen sind, den Implementierungsaufwand der Servicemodelle durchschnittlich mit einem Wert von 4,42 am höchsten einschätzen. Lediglich bei den Servicemodellen Ferndiagnose und Uptime-Garantie ist dies nicht der Fall. Dies kann damit erklärt werden, dass die Unternehmen oftmals mit dem kontinuierlichen Sammeln von Nutzungsdaten starten, um daraus Implikationen für die prädiktive Wartung, wozu auch die Ferndiagnose gehört, abzuleiten (Voigt und Steinmann 2013). Wenn ein Unternehmen genug Daten und Erfahrung mit der Ferndiagnose hat, sind die Voraussetzungen gegeben, um eine Uptime-Garantie für die entsprechenden Maschinen anbieten zu können (Suppatvech et al. 2019). Daher ist plausibel, dass die Uptime-Garantie insbesondere für Maschinenbauer eine erstrebenswerte Basis für neue, digitale Geschäftsmodelle sein kann, was sich auch in der im Vergleich zu den anderen Branchen hohen Realisierungswahrscheinlichkeit niederschlägt.

Unternehmen, die im Anlagenbau und der Bereitstellung von Systemlösungen tätig sind, beurteilen den Implementierungsaufwand für Ferndiagnose und UptimeGarantie noch höher als die Maschinenbauer. Vor dem Hintergrund, dass die entsprechenden Geschäftsmodelle im Anlagenbau meist projektspezifisch und einmalig zu entwickeln sind, während im Maschinenbau aufgrund der häufigeren Serienfertigung eher eine Mehrfachverwendbarkeit der entwickelten Dienste anzutreffen ist, erscheint dies plausibel. Ebenfalls nachvollziehbar ist, dass Anlagenbauer im Management der Anlagen für den Kunden ein im Branchenvergleich sehr attraktives Servicemodell sehen, da der optimale Betrieb von Systemlösungen regelmäßig spezifische Dienste des Anlagenbauers erfordert.

Bei Unternehmen der Elektroindustrie fällt auf, dass diese besonders das Servicemodell Digital Add-on positiv einschätzen. Dies manifestiert sich in einem hohen Mehrwert, niedrigem Implementierungsaufwand und einer daraus resultierenden relativ hohen Realisierungswahrscheinlichkeit. Dies ist nachvollziehbar, da Digital Add-on vor allem im Zusammenhang mit weniger komplexen Produkten oder Komponenten, bei denen das Augenmerk des Kunden nicht auf die Gesamtleistung fokussiert ist, seine Wirkung als digitales Geschäftsmodell entfalten kann.

Wie oben beschrieben, sehen Metallerzeuger und -bearbeiter einen signifikant geringeren Nutzen und eine niedrigere Realisierungswahrscheinlichkeit in fast allen beschriebenen datenbasierten Servicemodellen. Die Ausnahme stellt das Modell Qualitätsmanagement dar. Hier sehen die Metall-Unternehmen eine deutlich höhere Realisierungswahrscheinlichkeit als ihre Kollegen in anderen Branchen. Ein Grund für diese Erkenntnis könnte in der Natur der Produkte der Branche liegen: Diese stehen oft am Anfang der Wertschöpfungskette der Kunden, weshalb ein besonderes Augenmerk auf die Dokumentation der Qualität der Eingangswerkstoffe gelegt wird. 
Auch bei den Servicemodellen Solution Provider und Ersatzteilanzeige wurde der Implementierungsaufwand als relativ hoch eingestuft, was allerdings nicht zu einer durchschnittlich tieferen Realisierungswahrscheinlichkeit geführt hat. Dies dürfte am doch sehr hohen Nutzen liegen, der dazu führt, dass größere Unternehmen durchaus willens sind, Implementierungshürden mit entsprechendem Aufwand aus dem Weg zu räumen.

Gleichwohl ist zu bemerken, dass die Absolutwerte für die Realisierungswahrscheinlichkeiten innerhalb von drei Jahren mit 2,4 bis maximal 3,9 auf der Skala von 1 bis 6 relativ niedrig sind. In Anbetracht des doch erkannten Nutzens deutet das darauf hin, dass zwar eine Umsetzung angestrebt wird, drei Jahre hierfür allerdings eine zu kurze Zeitskala sein könnten. Dies mag einerseits daran liegen, dass viele der befragten Unternehmen den Druck durch die Digitalisierung auf ihre etablierten Geschäftsmodelle noch nicht als kurzfristig bedrohlich wahrnehmen. Andererseits kann vermutet werden, dass sowohl die Schaffung der technischen Voraussetzungen als auch die tatsächliche Umstellung eines Geschäftsmodells Aufgaben sind, die in der Unternehmenspraxis oft mehr als drei Jahre in Anspruch nehmen.

\section{Fazit}

KMU der MEM-Branche (Maschinen-, Anlagen-, Elektro- und Metallindustrie) haben scheinbar verstanden, dass die Entwicklung neuer, digitaler Geschäftsmodelle im Zeitalter des IoT hochrelevant ist, um sich gegenüber Wettbewerbern zu differenzieren, die Kundenbindung zu verbessern und um höhere Margen als mit dem reinen Verkauf von Produkten wie Maschinen, Anlagen, Geräte und Komponenten zu erzielen. Datenbasierte, auf IoT-Komponenten und digitalisierten Prozessen beruhende Dienstleistungen stellen hierbei eine wichtige Grundlage dar.

Grundsätzlich lässt sich festhalten, dass die in dieser Studie identifizierten datenbasierten Servicemodelle einen moderaten bis hohen Mehrwert für KMU der MEMBranche aufweisen, da sie im Durchschnitt über alle Branchen eine Beurteilung von 3,75 bis 4,8 auf der Skala von 1 bis 6 erreichen. Die breite Masse der Befragten sieht dabei den größten Nutzen in den Servicemodellen Ferndiagnose, Solution Provider und Ersatzteilanzeige. Dies mag zum einen daran liegen, dass in der MEM-Branche bereits seit einigen Jahren technisch ausgereifte und nachweislich erfolgreiche Geschäftsmodelle für diese datenbasierten Services bestehen. Dies ist speziell bei der Ferndiagnose der Fall, womit sich auch die für dieses Servicemodell ermittelten niedrigen Implementierungshürden bzw. hohen Realisierungswahrscheinlichkeiten erklären lassen.

Unter Umständen wiegt bereits der unternehmensinterne Nutzen eines datenbasierten Servicemodelles (beispielsweise Transparenz über die kundenseitige Nutzungsdauer und -intensität eines Produktes) bereits den damit verbundenen Implementierungsaufwand auf. Dennoch wäre es aus Unternehmenssicht wünschenswert, wenn durch ein datenbasiertes Servicemodell auch für den Kunden ein Nutzen entsteht, für den eine Zahlungsbereitschaft existiert.

Hinsichtlich der bevorzugten Vergütungsmodelle konnte bei den meisten Servicemodellen keine klare Präferenz gefunden werden. Dies könnte darauf zurückzufüh- 
ren zu sein, dass die Wahl der Vergütungsform sehr stark von Branchen bzw. von Branchensegmenten sowie der Kundenbeziehung abhängt. Um die Monetarisierung zu optimieren, müsste man die Akzeptanz der Zielkunden für die verschiedenen Vergütungsmodelle individuell untersuchen.

Zusammenfassend lässt sich festhalten, dass datenbasierte Servicemodelle für alle Unternehmen der MEM-Branche einen hohen Mehrwert aufweisen können, die Realisierungswahrscheinlichkeit in den nächsten drei Jahren jedoch nur als moderat eingeschätzt wird. Unternehmen, die entsprechende Services einführen möchten, sollten daher nebst dem Mehrwert den erwarteten Implementierungsaufwand genauer abschätzen, da dieser je nach gewähltem Modell sehr unterschiedlich ausfallen kann.

Die Identifizierung von Faktoren, welche die Umsetzung von datenbasierten Services und den damit verbundenen digitalen Geschäftsmodellen ermöglichen, bergen ein großes Potenzial und Bedarf für weitere Forschung.

Funding Open access funding provided by ZHAW Zurich University of Applied Sciences

Open Access Dieser Artikel wird unter der Creative Commons Namensnennung 4.0 International Lizenz veröffentlicht, welche die Nutzung, Vervielfältigung, Bearbeitung, Verbreitung und Wiedergabe in jeglichem Medium und Format erlaubt, sofern Sie den/die ursprünglichen Autor(en) und die Quelle ordnungsgemäß nennen, einen Link zur Creative Commons Lizenz beifügen und angeben, ob Änderungen vorgenommen wurden.

Die in diesem Artikel enthaltenen Bilder und sonstiges Drittmaterial unterliegen ebenfalls der genannten Creative Commons Lizenz, sofern sich aus der Abbildungslegende nichts anderes ergibt. Sofern das betreffende Material nicht unter der genannten Creative Commons Lizenz steht und die betreffende Handlung nicht nach gesetzlichen Vorschriften erlaubt ist, ist für die oben aufgeführten Weiterverwendungen des Materials die Einwilligung des jeweiligen Rechteinhabers einzuholen.

Weitere Details zur Lizenz entnehmen Sie bitte der Lizenzinformation auf http://creativecommons.org/ licenses/by/4.0/deed.de.

\section{Literatur}

Acatech (2014) Smart Service Welt - Umsetzungsempfehlungen für das Zukunftsprojekt Internetbasierte Dienste für die Wirtschaft. Acatech Working Paper

Baines T, Lightfoot H, Evans S et al (2007) State-of-the-art in product-service systems. Proc Inst Mech Eng B J Eng Manuf 221:1543-1552

Bauernhansl T, Emmrich V, Döbele M, Paulus-Rohmer D, Schatz A, Weskamp M (2015) GeschäftsmodellInnovation durch Industrie 4.0 - Chancen und Risiken für den Maschinen- und Anlagenbau. Dr. Wieselhuber \& Partner GmbH und Fraunhofer IPA, München

BMWi (2018) Innovationstreiber für digitale Geschäftsmodelle. https://www.plattform-i40.de/PI40/ Redaktion/DE/Downloads/Publikation/innovationstreiber-digitaler-geschaeftsmodelle.html. Zugegriffen: 1. Juni 2020

Davenport T, Westerman G (2018) Why so many high-profile digital transformations fail. https://hbr. org/2018/03/why-so-many-high-profile-digital-transformations-fail. Zugegriffen: 20. Mai 2020 (Harvard Business Review)

Fleisch E, Weinberger M, Wortmann F (2015) Geschäftsmodelle im Internet der Dinge. Schmalenbachs Z Betriebswirtsch Forsch 67:444-465

Friedli T, Gebauer H (2003) Erfolgsfaktoren für ein professionelles Dienstleistungsmanagement in produzierenden Unternehmen. Industrie-Management 19:74-77 
Fuchs R, Barth L (2018) Wie Smart Connected Products Kunden emotionalisieren. In: Rüeger BP, Hannich F, Fuchs R et al (Hrsg) Emotionalisierung im digitalen Marketing: Erfolgreiche Methoden für die Marketingpraxis. Schäffer-Poeschel, Stuttgart, S 89-103

Gassmann O, Frankenberger K, Csik M (2013) Geschäftsmodelle entwickeln: 55 innovative Konzepte mit dem St. Galler Business Model Navigator. Carl Hanser, München

Gerpott T, May S (2016) Integration of Internet of Things components into a firm's offering portfolio-A business development framework. Emerald Insight 18:53-63

Haller S, Karnouskos S, Schroth C (2008) The internet of things in an enterprise context. In: Domingue J, Fensel D, Traverso P (Hrsg) Future Internet Symposium. Springer, Berlin/Heidelberg, S 14-28

Herterich M, Uebernickel F, Brenner W (2015) The next wave of service innovation: how cyber-physical systems can be leveraged for effective industrial equipment operations and empower industrial service. University of St.Gallen Working Paper

Jaekel M (2015) Die Anatomie digitaler Geschäftsmodelle. Springer Vieweg, Wiesbaden

Krebs A, Hepp M, Hadwich K (2017) Erfolgsfaktoren der Integration wissensintensiver Dienstleistungen im Rahmen der Servicetransformation. In: Bruhn M, Hadwich K (Hrsg) Dienstleistungen 4.0. Springer Gabler, Wiesbaden, S 133-156

Lightfoot H, Baines T, Smart P (2013) The servitization of manufacturing: a systematic literature review of interdependent trends. Int J Oper Prod Manag 33:1408-1434

Müller JM, Buliga O, Voigt K-I (2018) Technological forecasting and social change fortune favors the prepared: how SMEs approach business model innovations Industry 4.0. Technol Forecast Soc Change 132:2-17

Neely A (2008) Exploring the financial consequences of the servitization of manufacturing. Oper Manag Res 2:103-118

Oliva R, Kallenberg R (2003) Managing the transition from products to services. Int J Serv Ind Manag 14:160-172

Osterwalder A, Pigneur Y (2010) Business model generation: a handbook for visionaries, game changers and challengers. John Wiley \& Sons, Hoboken

Paukstadt U, Strobel G, Eicker S, Becker J (2019) Von smarten Produkten zu smarten Dienstleistungen und deren Auswirkung auf die Wertschöpfung. HMD 56:494-513

Porter M, Heppelmann J (2014) How smart, connected products are transforming competition. Harv Bus Rev 92:64-88

Roy R, Shebab E, Tiwari A, Baines T, Lightfoot H, Benedettini O, Kay J (2009) The servitization of manufacturing-A review of literature and reflection on future challenges. J Manuf Technol Manag 5:547-567

Rymaszewska A, Helo P, Gunasekaran A (2017) IoT powered servitization of manufacturing-An exploratory case study. Int J Prod Econ 192:92-105

Schallmo D (2013) Geschäftsmodelle erfolgreich entwickeln und implementieren. Springer Gabler, Wiesbaden

Scherrer M, Deflorin P, Schillo K, Ziltener A (2017) Business model innovation triggered by Industry 4.0. In: Spring Servitization Conference Luzern

Schneider P (2016) Welche Auswirkungen hat Industrie 4.0 auf KMU? Das Geschäftsmodell als Analyseinstrument. Z KMU Entrepreneursh 64:253-279

Suppatvech C, Godsell J, Day S (2019) The roles of internet of things technology in enabling servitized business models: a systematic literature review. Ind Mark Manag 82:70-86

Swissmem (2018) Umsetzungsstand Industrie 4.0 - Umfrage in der Schweizer MEM-Branche 2018. https://www.swissmem.ch/fileadmin/user_upload/Swissmem_Umfrage_Umsetzungsstand_Industrie4_ 2018.pdf. Zugegriffen: 2. Mai 2020

Vogel-Heuser B (2017) Herausforderungen und Anforderungen aus Sicht der IT und der Automatisierungstechnik. In: Vogel-Heuser B, Bauernhansl T, Ten Hompel M (Hrsg) Handbuch Industrie 4.0. Springer Vieweg, Berlin/Heidelberg, S 33-44

Voigt K-I, Steinmann F (2013) Condition monitoring as a key technology-An analysis of requirements for new business models for remote services. In: Symposium für Vorausschau und Technologieplanung Berlin

Wortmann F, Bilgeri D, Gebauer H, Lamprecht C, Fleisch E (2019) Geld verdienen im IoT - aber wie? HMD 56:1094-1112 